\title{
Measuring the chemical composition of cosmic rays with AMS
}

\author{
R.J. García López ${ }^{1,2}$, C. Delgado ${ }^{1}$, M. Panniello ${ }^{1}$, M.T. Costado $^{1,2} \&$ \\ J. Calvo ${ }^{1}$ for the AMS Collaboration \\ ${ }^{1}$ Instituto de Astrofísica de Canarias, C/ Vía Láctea s/n, E-38205 La Laguna, Tenerife, Spain \\ ${ }^{2}$ Departamento de Astrofísica, Universidad de La Laguna \\ Av. Astrofísico Francisco Sánchez s/n, E-38206 La Laguna, Tenerife, Spain \\ email:\{rgl, delgadom, maurizio, mcostado, jct $\} @$ iac.es
}

\begin{abstract}
AMS (Alpha Magnetic Spectrometer) is a particle detector designed to operate at the International Space Station. Starting in 2008, its purpose is to perform accurate, high statistics, long duration measurements of energetic $(0.1 \mathrm{GeV}$ to $\sim \mathrm{TeV})$ charged cosmic ray spectra in space, providing fundamental information about key ingredients in the spallation reactions taking place in the interstellar medium. We present here the characteristics of this experiment and the extense collaboration supporting the project.
\end{abstract}

Keywords. Instrumentation: detectors, instrumentation: spectrographs, space vehicles: instruments, cosmic rays

\section{AMS experiment.}

AMS is a particle physics experiment in space. Its purpose is to perform accurate, high statistics, long duration direct measurement of the spectra of energetic (up to multi-TeV) primary charged cosmic rays. The detector will be installed in the International Space Station by the beginning of 2008 and will work during, at least, three years (The AMS Collaboration 2005). Some of the physics goals are:

- Dark Matter: There are many theoretical suggestions that particles predicted by Super-Symmetry (SUSY) theories are a component of the Dark Matter (Jungman, Kamionkowski \& Griest 1996. Annihilation of dark matter in the Galactic halo produces anti-protons, positrons and gammas. The precision measurement of the spectrum of these species in the cosmic rays will enable us to establish whether SUSY particles are the origin of Dark Matter.

- Antimatter: The strong evidence which supports the Big Bang theory requires matter and antimatter to be equally abundant at the very hot beginning. The absence of large quantities of antimatter within our cluster of galaxies is well established (Cohen, Rújula \& Glashow 1998). However there is not direct evidence regarding the remainder of the universe. Thus the search for isolated antinuclei within the bulk of cosmic rays could settle down the question of the existence of large antimatter domains.

- Cosmic rays: AMS will collect $\sim 10^{9}$ nuclei and isotopes, which will allow to study several interesting issues in physics. Among them, understanding the propagation and origin of the cosmic rays will be a key goal.

To perform a high accuracy measurement of the spectra of energetic charged particles, AMS has been designed based on the experience and observations from experiments to study rare signals among intense backgrounds. These experiments were successful because their minimal material along the particle trajectory and their redundant measurement 
of momentum and velocity. It was the strict adherence to these techniques that ensured that the background rejection of $10^{10}$ necessary for AMS goals was indeed possible.

The construction of the AMS detector is being carried out by a worldwide collaboration of more than 56 institutes of 15 countries. The number of physicists involved in the experiment amounts to around 500. The detector contains the following main components:

- A Transition Radiation Detector (TRD), which identifies electrons and positrons with a measured rejection factor against hadrons of $10^{3}$ to $10^{2}$ from $1.5 \mathrm{GeV}$ to $300 \mathrm{GeV}$.

- Time of Flight (TOF) hodoscopes, to measure velocity and energy deposition.

- A superconducting magnet, which provides a larger bending power for charged particles.

- A large area $\left(6.45 \mathrm{~m}^{2}\right)$ silicon tracker which provides a proton rigidity (三momentum/ charge) resolution of $20 \%$ at $0.5 \mathrm{TeV}$ and charge measurement up to iron $(\mathrm{Z}=26)$.

- A Ring Imaging Cherenkov Counter (RICH) which measures the velocity (better than $0.1 \%$ ) and charge of particles or nuclei.

- A 3-D sampling calorimeter (ECAL), which measures the energy of gamma rays, electrons and positrons, and provides a rejection of hadrons of $10^{4}$ in the energy range $1.5 \mathrm{GeV}$ to $14 \mathrm{TeV}$.

AMS will measure light element spectra in a wide energy range, improving accuracy and statistics as compared with previous experiments. In particular, it will identify $\sim 10^{4}$ $\mathrm{B}$ nuclei with $\mathrm{E}<100 \mathrm{GeV} / \mathrm{n}$ and $\sim 10^{5}{ }^{10}$ Be after 3 years, and hence determine the $\mathrm{B} / \mathrm{C}$ ratio for $\mathrm{E} \lesssim 1 \mathrm{TeV}$ and separate ${ }^{10} \mathrm{Be}$ from ${ }^{9} \mathrm{Be}$ in the energy range $0.15-10 \mathrm{GeV} / \mathrm{n}$

\section{Ring Imaging Cherenkov Counter (RICH).}

AMS goals require to have a precise determination of the masses of charged particles. The mass can be inferred through the determination of the momentum and velocity. In AMS spectrometer, the momentum is determined by the Tracker with a relative accuracy of $\sim 1 \%$ over a wide energy range. This entails an error of the same order on the mass of the particle so, in order to overcome this uncertainty, the velocity has to be measured with a relative accuracy of about 1 per mil. For this purpose a RICH detector with a large geometrical acceptance has been designed. The measurement of the velocity of the charged particles is based on the Cherenkov effect: when a charged particle goes through a dielectric material with a velocity larger than the phase velocity of electromagnetic fields in the material, the change of the dipolar field with time initiates the emission of electromagnetic radiation. The characteristics of this emission are directly related to the velocity and charge of the particle and the refractive index of the material.

The RICH detector has a truncated conical shape and is placed below the lower TOF planes and above the ECAL detector. A charged particle coming from the top of the AMS detector crosses an aerogel-NaF radiator creating a cone of Cherenkov radiation. This radiation is detected in a plane instrumented with 680 multianode photomultipliers. From the measured shape on the radiation cone and its intensity, the velocity and charge of the particle are inferred.

The RICH is being built by a collaboration of 6 institutes spread over 5 countries: the mechanic design and construction is done by INFN-Bologna (Italy), IAC (Spain) and CIEMAT (Spain); the electronics are built at LPSC (France) and CIEMAT; finally, LIEPP of U. of Maryland (USA), LIP (Portugal), IAC and CIEMAT are in charge of the reconstruction, simulation and analysis software.

In order to validate the design of this detector a prototype, roughly equivalent to one eighth of the final detector, has been tested on a beam of ions. The analysis of the 
obtained data shows that RICH performance is close to the design goals: the velocity resolution for protons is better than 1 per mil, and it improves with particle charge; the charge determination resolution is enough to distinguish the different elements above iron $(\mathrm{Z}=26)$. The test beam results have been used to implement an accurate simulation of the final detector response. That has been used to study the expected AMS-RICH isotopic and chemical identification of cosmic rays performance (see details about the identification performance in Mercedes Mollá in this volume).

\section{References}

The AMS Collaboration 2005, AMS on ISS. Construction of a partilce physics detector on the Internation Space Station. To be published in Nucl. Instr. Methods A.

Jungman G., Kamionkowski M. \& Griest K. 1996, Physics Reports 267, 195

Cohen A., Rújula A. \& Glashow S. 1998, Astrophysical Journal 495, 539 


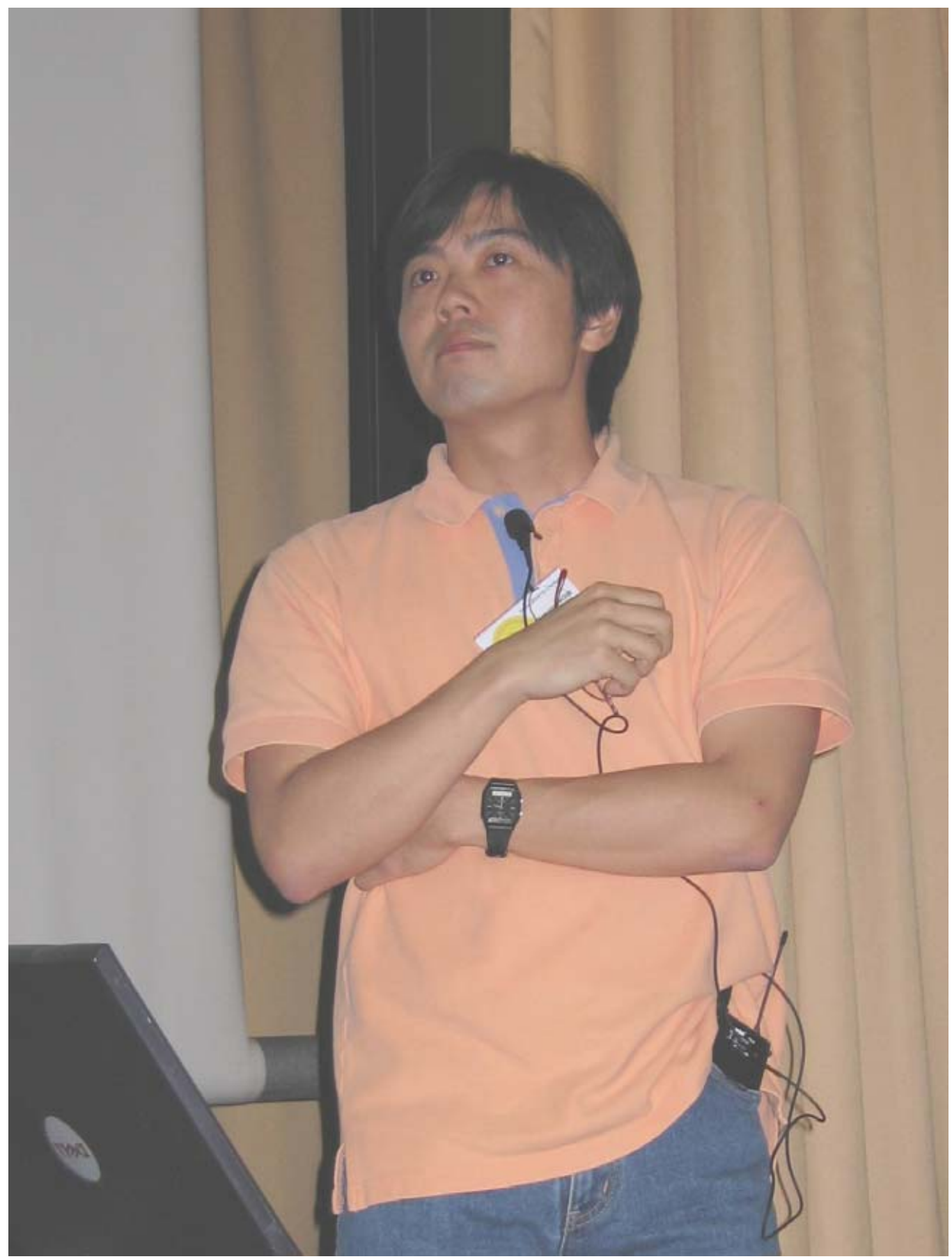

Susumu Inoue, answering questions at the end of his talk about ${ }^{6} \mathrm{Li}$. 